Research Article

\title{
New Traveling Wave Solutions and Interesting Bifurcation Phenomena of Generalized KdV-mKdV-Like Equation
}

\author{
Yiren Chen ${ }^{1}$ and Shaoyong $\mathrm{Li} \mathbb{D}^{2}$ \\ ${ }^{1}$ College of Mathematics and Statistics, Shenzhen University, Shenzhen 518060, China \\ ${ }^{2}$ School of Mathematics and Statistics, Shaoguan University, Shaoguan 512005, China \\ Correspondence should be addressed to Shaoyong Li; lishaoyongok@qq.com
}

Received 18 June 2021; Revised 5 August 2021; Accepted 7 November 2021; Published 20 November 2021

Academic Editor: Mohammad Mirzazadeh

Copyright (C) 2021 Yiren Chen and Shaoyong Li. This is an open access article distributed under the Creative Commons Attribution License, which permits unrestricted use, distribution, and reproduction in any medium, provided the original work is properly cited.

\begin{abstract}
Using the bifurcation method of dynamical systems, we investigate the nonlinear waves and their limit properties for the generalized $\mathrm{KdV}$-mKdV-like equation. We obtain the following results: (i) three types of new explicit expressions of nonlinear waves are obtained. (ii) Under different parameter conditions, we point out these expressions represent different waves, such as the solitary waves, the 1-blow-up waves, and the 2-blow-up waves. (iii) We revealed a kind of new interesting bifurcation phenomenon. The phenomenon is that the 1-blow-up waves can be bifurcated from 2-blow-up waves. Also, we gain other interesting bifurcation phenomena. We also show that our expressions include existing results.
\end{abstract}

\section{Introduction}

Most relationships in nature and human society are intrinsically nonlinear rather than linear in nature, so many phenomena in nature and human society can be described by nonlinear equations, such as automatic control, meteorology, engineering calculation, engineering budget, economy, and finance $[1,2]$. Nowadays, many scientists are very interested in nonlinear equations and their solutions and have done a lot of related work [3-5].

In the paper, we consider the generalized KdV-mKdVlike equation $[6,7]$.

$$
u_{t}+\left(\alpha+\beta u^{p}+\gamma u^{2 p}\right) u_{x}+u_{x x x}=0
$$

where $p>0, \alpha, \beta, \gamma \neq 0$ are real constants. By using appropriate parameters, the generalized $\mathrm{KdV}$-mKdV-like equation becomes the classical KdV equation [8-11], the mKdV equation [12-16], the KdV-like equation [17-20], and the generalized $\mathrm{mKdV}$ equation [21].

Up to now, many authors have been interested in the study of the many forms of $\mathrm{KdV}$-like equations [22-25], and there are several explicit solutions results of the general- ized KdV-mKdV-like equation based on the significant physical background. For example, Li and Wang [6] gave the following traveling wave solution:

$$
u_{w}(\xi)=\left[\frac{-2(p+1)(p+2)(2 p+1) \beta}{(2 p+1) \beta^{2}(p \xi)^{2}+(p+1)(p+2)^{2} \gamma}\right]^{1 / p},
$$

where $\beta<0, \gamma>0, \xi=x-\alpha$.

In recent years, the bifurcation method of dynamical systems has been widely used in investigating the nonlinear partial differential equations, for instance [26-29].

In this paper, we study the nonlinear wave solutions and the bifurcation phenomena for Eq. (1). First, we obtain three types of explicit waves which represent the solitary waves, the 1-blow-up waves, and the 2-blow-up waves. Second, we reveal the new bifurcation phenomena which are introduced in the abstract above. Furthermore, we obtain other interesting bifurcation phenomena. The first phenomenon is that the 1-blow-up waves can be bifurcated from the solitary waves. The second phenomenon is that the trivial waves can be bifurcated from the solitary waves.

This paper is organized as follows. In Section 2, we give some notations and state our main results. Our main 


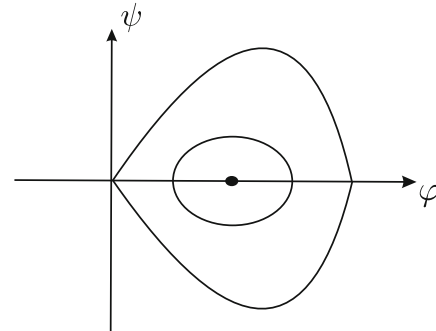

(a) $A_{1}: \beta<0, \gamma>0$

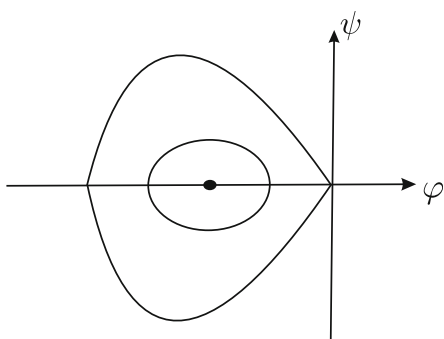

(c) $A_{2}: \beta>0, \gamma>0$

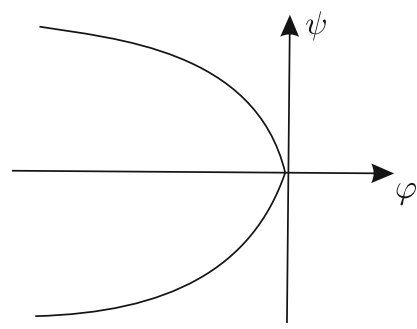

(e) $\beta>0, \gamma=0$

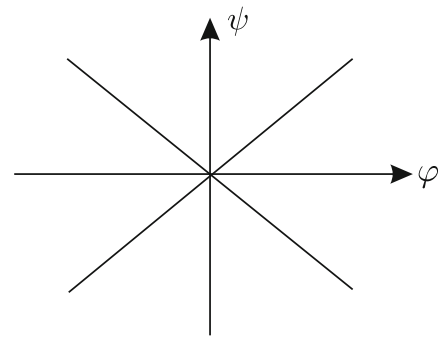

(g) $\beta=0, \gamma<0$

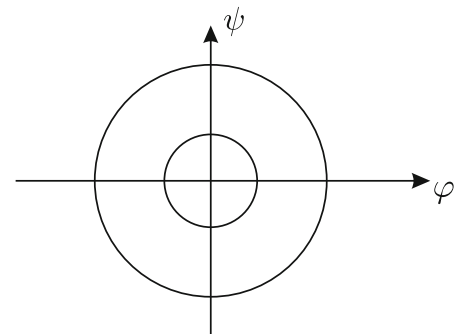

(b) $\beta=0, \gamma>0$

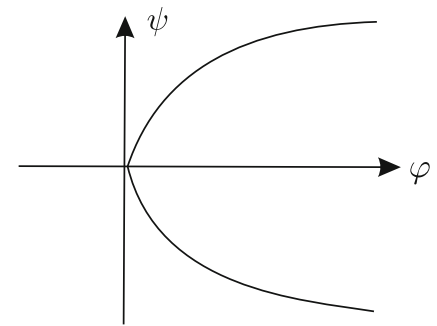

(d) $\beta<0, \gamma=0$

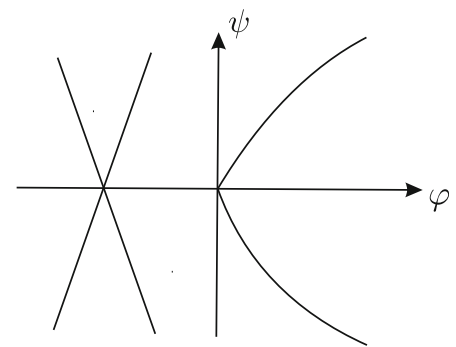

(f) $A_{3}: \beta<0, \gamma<0$

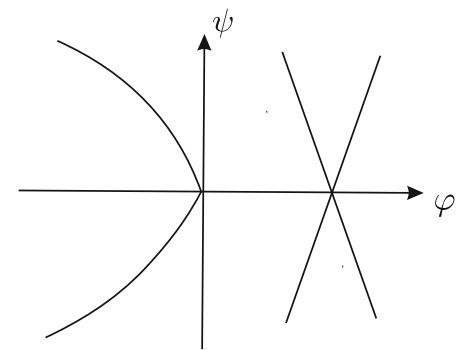

(h) $A_{4}: \beta>0, \gamma<0$

FIgURE 1: The phase portraits of the system (12).

derivations are listed in Section 3. A brief conclusion is given in Section 4.

\section{Our Main Results}

In this paper, $p$ is odd and the situation of even is similar to study. In this section, we state our main results. In order to state these results conveniently, we give some notations which will be used in the latter statement and the derivations.

The zones $A_{j}(j=1,2,3,4)$ are given in Figure 1 , and $\kappa$ is an arbitrary real constant. In this article, we only consider the case $\alpha-c=0$. For other cases, due to the complexity, we will investigate them in our future works.
Proposition 1. If $\alpha-c=0$, then, the explicit solutions are

$$
u_{1}(\xi)=\left(\frac{-2(p+1)(p+2)(2 p+1) \beta}{(2 p+1) \beta^{2}(p \xi+\kappa)^{2}+(p+1)(p+2)^{2} \gamma}\right)^{1 / p}
$$

and 


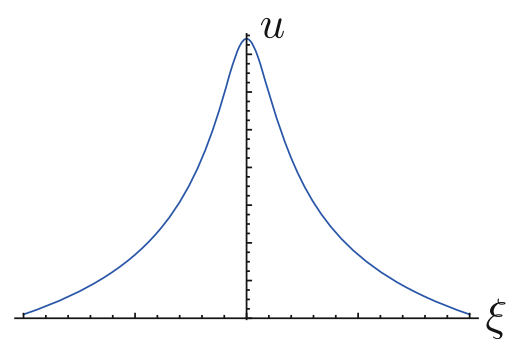

(a) $\gamma=10^{-1}$

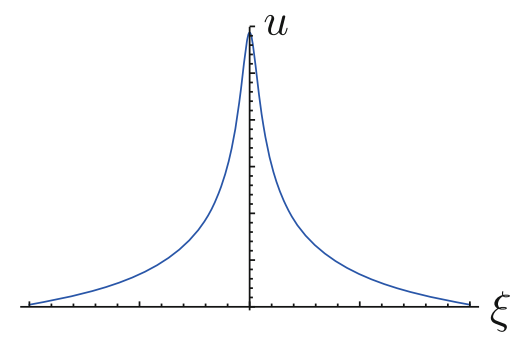

(b) $\gamma=10^{-2}$

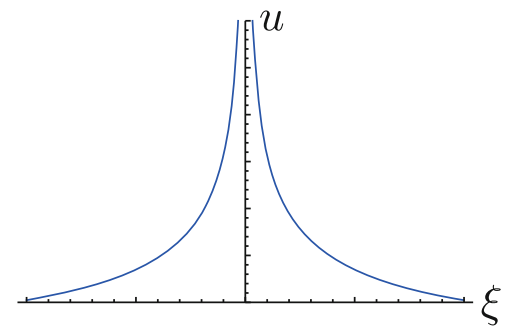

(c) $\gamma=10^{-4}$

FIGURE 2: The varying figures of the example of $u=u_{1}(\xi)$ when $\kappa=0, p=5, \alpha-c=0, \beta=-3$, and $\gamma \longrightarrow 0+0$.

when $\kappa=0, u_{1}$ becomes

$$
u_{1}^{0}(\xi)=\left(\frac{-2(p+1)(p+2)(2 p+1) \beta}{(2 p+1)(\beta p \xi)^{2}+(p+1)(p+2)^{2} \gamma}\right)^{1 / p}
$$

After selecting the appropriate parameters, $u_{1}^{0}$ is equivalent to $u_{w}$.

when $\gamma=0, u_{1}$ becomes

$$
u_{1}^{1}(\xi)=\left(\frac{-2(p+1)(p+2)}{\left.\beta(p \xi+\kappa)^{2}\right)}\right)^{1 / p}
$$

(i) If $(\beta, \gamma) \in A_{1}$ or $A_{2}$, then, $u_{1}$ is symmetric solitary wave (the example is given in Figure 2(a) or Figure 3(a)). Specially, when $\gamma \longrightarrow 0+0$, then, the symmetric solitary wave $u_{1}$ becomes single-side 1blow-up wave $u_{1}^{1}$ (the example is given in Figure 2(c)), and for the varying process of the example, see Figure 2. When $\beta \longrightarrow 0 \pm 0$, then, the symmetric solitary wave $u_{1}$ becomes the trivial wave (the example is given in Figure 3(c)), and for the varying process of the example, see Figure 3

(ii) If $(\beta, \gamma) \in A_{3}$ or $A_{4}$, then, $u_{1}$ is 2-blow-up solitary wave (the example is given in Figure 4(a)). Specially, when $\gamma \longrightarrow 0-0$, then, the 2-blow-up wave $u_{1}$ becomes the single-side 1-blow-up wave $u_{1}^{1}$ (the example is given in Figure 4(c)), and for the varying process of the example, see Figure 4

(iii) If $\beta=0, \gamma<0$, then, $u_{2}$ is 1 -blow-up solitary wave

\section{The Derivation of Main Results}

To derive our results, we give some preliminaries in this section. For simplicity of the derived expression, we use the following notation

$$
\begin{gathered}
A=\frac{\gamma}{2(p+1)(2 p+1)}, \\
B=\frac{\beta}{(p+1)(p+2)}, \\
C=\frac{\alpha-c}{2} .
\end{gathered}
$$

then we derive our main results.

3.1. The Derivations to Proposition 1. For given constant $c$ and $c-\alpha=0$, substituting $u=\varphi(\xi)$ with $\xi=x-c t$ into Eq.(1), it follows that

$$
\beta \varphi^{p} \varphi^{\prime}+\gamma \varphi^{2 p} \varphi^{\prime}+\varphi^{\prime \prime \prime}=0
$$

Integrating (10) once and letting the integral constant be zero, we get

$$
\frac{\beta}{p+1} \varphi^{p+1}+\frac{\gamma}{2 p+1} \varphi^{2 p+1}+\varphi^{\prime \prime}=0
$$

Letting $\psi=\varphi^{\prime}$, we obtain a planar system

$$
\left\{\begin{array}{l}
\frac{\mathrm{d} \varphi}{\mathrm{d} \xi}=\psi, \\
\frac{\mathrm{d} \psi}{\mathrm{d} \xi}=-\frac{\gamma}{2 p+1} \varphi^{2 p+1}-\frac{\beta}{p+1} \varphi^{p+1}
\end{array}\right.
$$




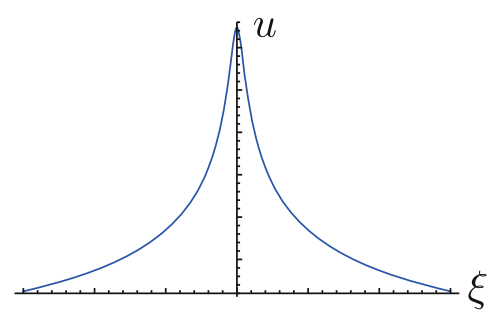

(a) $\gamma=10^{-1}$

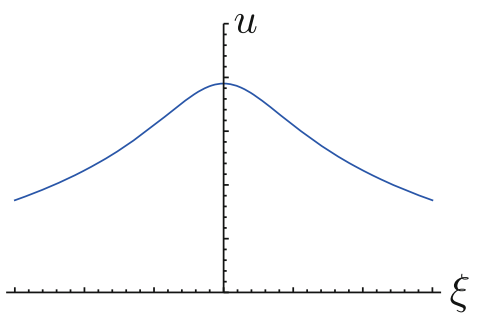

(b) $\gamma=10^{-2}$

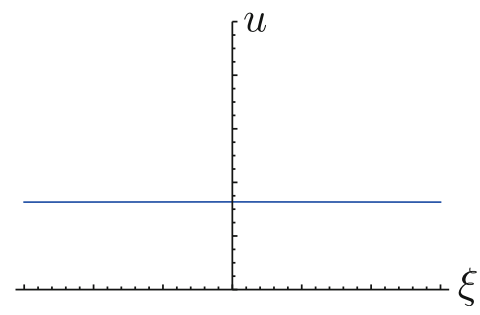

(c) $\gamma=10^{-4}$

FIgURE 3: The varying figures of the example of $u=u_{1}(\xi)$ when $\kappa=0, p=9, \alpha-c=0, \gamma=1$, and $\beta \longrightarrow 0-0$.

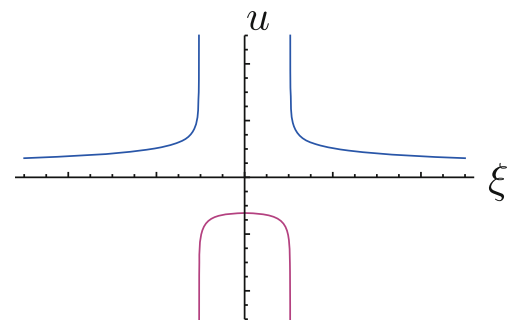

(a) $\gamma=10^{-1}$

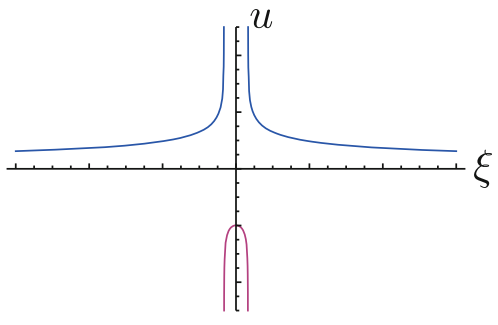

(b) $\gamma=10^{-2}$

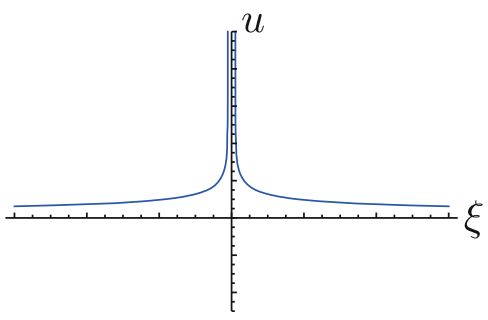

(c) $\gamma=10^{-4}$

FIgURE 4: The varying figures of the example of $u=u_{1}(\xi)$ when $\kappa=0, p=9, \alpha-c=0, \gamma=1$, and $\beta \longrightarrow 0-0$.

with the first integral

$$
H(\varphi, \psi)=\frac{1}{2} \psi^{2}+\frac{\gamma}{2(p+1)(2 p+1)} \varphi^{2 p+2}+\frac{\beta}{(p+1)(p+2)} \varphi^{p+2}=h,
$$

where $h$ is the integral constant. According to the qualitative theory, we obtain the bifurcation phase portraits of system (12) as Figure 1. By means of the bifurcation phase portraits, we can derive Proposition 1.

In the first integral (13), letting $h=H(0,0)$, we obtain

$$
\psi^{2}=-2 \varphi^{2}\left(A \varphi^{2 p}+B \varphi^{p}\right)
$$

Substituting (14) into the first equation of (12) and inte- grating it, we get

$$
\int_{l}^{\varphi} \frac{d s}{s \sqrt{A s^{2 p}+B s^{p}}}=|\xi|
$$

where $l$ is an arbitrary constant or $\pm \infty$.

When $\beta \neq 0$ and completing the integral above and solving the equation for $\varphi$, it will follow that

$$
\varphi=\left(\frac{-2(p+1)(p+2)(2 p+1) \beta}{(2 p+1) \beta^{2}(p \xi+\kappa)^{2}+(p+1)(p+2)^{2} \gamma}\right)^{1 / p},
$$

and letting $\kappa=0$, we can obtain (5) from (3). Similarly, when $\beta=0$ and completing the integral above and solving the equation for $\varphi$, we gain (4). Therefore, we have completed the derivations for Proposition 1. 


\section{Conclusion}

In this paper, we have investigated the explicit expressions of the nonlinear waves and their bifurcations in Eq. (1).

First, we obtained three types of new expressions. And they represent different waves, such as the solitary waves, the 1-blow-up waves, and the 2-blow-up waves.

Second, we revealed three kinds of bifurcation phenomena which include a new bifurcation phenomena. The first phenomenon which is new bifurcation phenomenon is that 1-blow-up waves can be bifurcated from 2-blow-up waves. The second phenomenon is that the trivial waves can be bifurcated from the solitary waves. The third phenomenon is that the 1-blow-up waves can be bifurcated from the solitary waves.

Third, we showed that a previous result is our special case, that is, $u_{w}$ is included in $u_{1}^{0}$.

Furthermore, the bifurcation method of dynamical systems can be used to find the new traveling solutions and bifurcations of many nonlinear equations such as the extended quantum Zakharov-Kuznetsov equation [37], the Fujimoto-Watanabe equation [38], and b-family-like equation [39]. We will continue to use the bifurcation method of dynamical systems to study other important nonlinear equations.

\section{Data Availability}

No data were used to support this study.

\section{Conflicts of Interest}

The authors declare that there is no conflict of interests regarding the publication of this article.

\section{Acknowledgments}

This work was supported by the National Natural Science Foundation of China Grant (12001377 and 11971176), the Outstanding Innovative Young Talents of Guangdong Province (2019KQNCX122), Natural Science Foundation of Guangdong Province (2018A0303100015), and Characteristic Innovative Project from Guangdong Provincial Department of Education (2018KTSCX204).

\section{References}

[1] X. P. Zhai and Y. R. Chen, "Global solutions and large time behavior for the chemotaxis-shallow water system," Journal of Differential Equations, vol. 275, pp. 332-358, 2021.

[2] Y. R. Chen, B. F. Feng, and L. M. Ling, "The robust inverse scattering method for focusing Ablowitz-Ladik equation on the non-vanishing background," Physica D: Nonlinear Phenomena, vol. 424, article 132954, 2021.

[3] L. Kaur and A. M. Wazwaz, "Bright - dark optical solitons for Schrodinger-Hirota equation with variable coefficients," Optik, vol. 179, pp. 479-484, 2019.

[4] I. Ahmed, A. R. Seadawy, and D. Lu, "M-shaped rational solitons and their interaction with kink waves in the Fokas-Lenells equation," Physica Scripta, vol. 94, no. 5, article 055205, 2019.
[5] S. Singh, L. Kaur, R. Sakthivel, and K. Murugesan, "Computing solitary wave solutions of coupled nonlinear Hirota and Helmholtz equations," Physica A: Statistical Mechanics and its Applications, vol. 560, article 125114, 2020.

[6] X. Z. Li and M. L. Wang, "A sub-ODE method for finding exact solutions of a generalized $\mathrm{KdV}-\mathrm{mKdV}$ equation with high-order nonlinear terms," Physics Letters A, vol. 361, no. 1-2, pp. 115-118, 2007.

[7] Z. L. Li, "Constructing of new exact solutions to the GKdV$\mathrm{mKdV}$ equation with any-order nonlinear terms by $\mathrm{G}^{\prime} \mathrm{G}$ expansion method," Applied Mathematics and Computation, vol. 217, no. 4, pp. 1398-1403, 2010.

[8] M. Song, X. J. Hou, and J. Cao, "Solitary wave solutions and kink wave solutions for a generalized KDV-mKDV equation," Applied Mathematics and Computation, vol. 217, no. 12, pp. 5942-5948, 2011.

[9] R. M. Miura, "The Korteweg-deVries equation: a survey of results," SIAM Review, vol. 18, no. 3, pp. 412-459, 1976.

[10] A. H. Khater, O. H. El-Kalaawy, and M. A. Helal, "Two new classes of exact solutions for the KdV equation via Backlund transformations," Chaos, Solitons \& Fractals, vol. 8, no. 12, pp. 1901-1909, 1997.

[11] M. Kovalyov and M. H. A. Abadi, "An explicit formula for a class of solutions of the KdV equation," Physics Letter A, vol. 254, no. 1-2, pp. 47-52, 1999.

[12] M. Lakshmanan and K. M. Tamizhmani, "Lie-Bäcklund symmetries of certain nonlinear evolution equations under perturbation around their solutions," Journal of Mathematical Physics, vol. 26, no. 6, pp. 1189-1200, 1985.

[13] L. R. T. Gardner, G. A. Gardner, and T. Geyikli, "Solitary wave solutions of the $\mathrm{MKdV}^{-}$equation," Computer Methods in Applied Mechanics and Engineering, vol. 124, no. 4, pp. 321333, 1995.

[14] B. Li, Y. Chen, and H. Q. Zhang, "Explicit exact solutions for compound KdV-type and compound KdV-Burgers-type equations with nonlinear terms of any order," Solitons \& Fractals, vol. 15, no. 4, pp. 647-654, 2003.

[15] J. Gorsky and A. Himonas, "Construction of non-analytic solutions for the generalized KdV equation," Journal of Mathematical Analysis and Application, vol. 303, no. 2, pp. 522-529, 2005.

[16] N. A. Kudryashov and D. I. Sinelshchikov, "A note on the lie symmetry analysis and exact solutions for the extended $\mathrm{mKdV}$ equation," Acta Applicandae Mathematicae, vol. 113, no. 1, pp. 41-44, 2011.

[17] B. Dey, "Domain wall solutions of KdV like equations with higher order nonlinearity," Journal of Physics A: Mathematical and General, vol. 19, no. 1, pp. L9-L12, 1986.

[18] B. Dey, "K-dV like equations with domain wall solutions and their Hamiltonians," Solitons, Springer Series in Nonlinear Dynamics, pp. 188-194, 1988.

[19] R. Grimshaw, D. Pelinovsky, E. Pelinovsky, and A. Slunyaev, "Generation of large-amplitude solitons in the extended Korteweg-de Vries equation," Chaos, vol. 12, no. 4, pp. 10701076, 2002.

[20] L. Zhengrong, J. Tianpei, Q. Peng, and X. Qinfeng, “Trigonometric function periodic wave solutions and their limit forms for the KdV-like and the PC-like equations," Mathematical Problems in Engineering, vol. 2011, Article ID 810217, 23 pages, 2011.

[21] Z. R. Liu and J. B. Li, "Bifurcations of solitary waves and domain wall waves for $\mathrm{KdV}$-like equation with higher order 
nonlinearity," International Journal of Bifurcation and Chaos, vol. 12, no. 2, pp. 397-407, 2002.

[22] K. Roy, A. Saha, P. Chatterjee, and C. S. Wong, "Head on collision of dust acoustic multi-solitons in a nonextensive plasma," Jurnal Fizik Malaysia, vol. 36, no. 1, pp. 0100901028, 2015.

[23] D. Lu, C. Yue, and M. Arshad, "Traveling wave solutions of space-time fractional generalized fifth-order KdV equation," Advances in Mathematical Physics, vol. 2017, Article ID 6743276, 6 pages, 2017.

[24] S. B. G. Karakoc, A. Saha, and D. Sucu, "A novel implementation of Petrov-Galerkin method to shallow water solitary wave pattern and superperiodic traveling wave and its multistability: generalized Korteweg-de Vries equation," Chinese Journal of Physics, vol. 68, pp. 605-617, 2020.

[25] B. Pradhan, A. Abdikian, and A. Saha, "Characteristics of supernonlinear and coexistence features for electron-acoustic waves in an adiabatic quantum plasma," The European Physical Journal D, vol. 75, no. 2, 2021.

[26] B. G. Zhang, W. B. Li, and X. P. Li, "Peakons and new exact solitary wave solutions of extended quantum ZakharovKuznetsov equation," Physics of Plasmas, vol. 24, no. 6, article 062113, 2017.

[27] Z. S. Wen, "Bifurcations and exact traveling wave solutions of the celebrated Green-Naghdi equations," International Journal of Bifurcation and Chaos, vol. 27, no. 7, article 1750114, 2017.

[28] S. K. El-Labany, W. F. El-Taibany, and A. Atteya, "Bifurcation analysis for ion acoustic waves in a strongly coupled plasma including trapped electrons," Physics Letters A, vol. 382, no. 6, pp. 412-419, 2018.

[29] J. P. Yang, R. Liu, and Y. R. Chen, "Bifurcations of solitary waves of a simple equation," International Journal of Bifurcation and Chaos, vol. 30, no. 9, article 2050138, 2020. 